\title{
DISCUSSION AFTER PAPER BY KRAFT
}

Baschek to Kraft: Is there any evidence of changes in the velocity field (of FG Sge), either shifts or different broadening of lines?

Kraft: There are some changes. The radial velocity is not quite constant. The observations are very difficult as it takes $8 \mathrm{~h}$ to get a coudé spectrum but I do not believe that the variations are surprising.

Paczyński to Kraft and Sackmann: I would like to point out that the properties of hydrogen and helium shell sources depend on the core mass and very little on the envelope mass. The same is true for the development of thermal pulses and the interpulse period. The difference between the models of Schwarzschild and Härm and those of Weigert was due to the difference of the core masses, $0.6 M_{\odot}$ and $0.8 M_{\odot}$ respectively. If you believe that the interpulse period for FG Sge was 6000 years, this indicates a core of $0.8 M_{\odot}$, but says nothing about the initial envelope mass. In any case the present envelope mass must be very small otherwise FG Sge would not be able to make an excursion to the hot part of the HR diagram. I understand that the carbon enrichment depends on the intershell mass. This in turn depends on the core mass only.

Kraft: I should like to caution everyone that values such as $6000 \mathrm{yr}$ must be regarded as very uncertain.

Schwarzschild to Kraft: I think we should congratulate Dr Kraft for the most important observations of FG Sge, which he has so perceptively and skillfully carried out. I suspect that it is very dangerous to overinterpret these new data rashly, however tempting. Nevertheless, I feel that we may be reasonably confident of two points. First, the timescale of the observed changes in radius strongly suggest that a shell flash is the basic cause of this remarkable phenomenon and, second, the rapid appearance of the $s$-process elements at the surface indicates that a shell flash can in fact produce these elements, as we had long expected on the basis of detailed flash computations. 